\title{
Autoimmunity in autism
}

\author{
Molecular Psychiatry (2002) 7, 329. DOI: 10.1038/ \\ $\mathrm{sj} / \mathrm{mp} / 4001137$
}

Autism was the topic of our last editorial. ${ }^{1}$ This focus continues. We will summarize findings on two new candidate systems: immune mediators as well as a neuropeptide (arginine vasopressin).

Our Image (page 334) and the article by Torrente et al (pages 375-382) show evidence of an immune-based enteropathy in autism. Those researchers describe extensive immunohistochemical analysis of small intestinal lesions in 25 children with regressive autism, 18 histologically normal controls, 11 children with celiac disease, and five children with cerebral palsy who required endoscopy for medical reasons. The authors report a novel form of immune enteropathy in autism, characterized by lymphocytic infiltration, increased crypt cell proliferation and enterocyte numbers with co-localization of IgG and complement $\mathrm{C} 1 \mathrm{q}$ on the enterocyte basolateral epithelial surface of 23/25 autistic children. This was not observed in the other groups. These findings raise the possibility of autoimmunity in severe autism.

As Torrente et al point out, the data could support different possibilities, such as the concept of local autoimmunity in the gut leading to altered cognitive functioning by failure to detoxify neuroactive substances originating from the gut. These findings would also be consistent with multisystem autoimmunity.

Several lines of evidence, well discussed in Torrente et al's article, would support an autoimmune basis for autism. Those include reports of association of major histocompatibility complex (MHC) genes with autism. The strongest associations between MHC genes and autism involve the null allele of complement C4B within the class III region, the extended haplotype B4S30-D4R, and the third hypervariable region of HLADR 1. These are all known to predispose to autoimmunity. There is also increased prevalence of autoimmune disorders in families of autistic children and two reports of circulating autoantibodies to brain components. Animal models are also revealing: several different strains of inbred mice with spontaneously occurring autoimmunity present with early neurobehavior abnormalities, which can be reversed by early treatment with cyclophosphamide.

Do these independent findings conclusively support the concept of autoimmunity in autism? There have been suggestions of autoimmune involvement in essentially all psychiatric disorders, including schizophrenia, depression and obsessive compulsive disorder, but the data do not yet support final conclusions. $^{2}$ The field of psychiatry is plagued with the issue of non-replication. Only after various independent replication reports appear will we be confident that the findings reported in this issue by Torrente et al are indeed relevant to autism. Another scenario may play itself out in the future. All diagnostic entities in psychiatry are essentially collections of symptoms without consideration of biological or etiological features. As our knowledge of the fundamental biology of psychiatric disorders evolves it is likely that what we now know as autism will be a collection of various diagnostic entities, each one with distinct pathophysiologic and pathogenic features, but sharing clinical presentations. This is common in medicine: anemia and meningitis consist of various distinct disorders with shared clinical features. It is possible that in the future there will be such a concept as 'autoimmune autism,' as a disorder within the autism spectrum. Other biological alterations may be the hallmarks of distinct disorders that may emerge from within our current classification of autism.

In our next issue we will publish an article by Ed Cook's group at The University of Chicago which would support the involvement of an additional candidate system in autism. That work attempts to connect years of work by the Insel laboratory demonstrating the role of arginine vasopressin receptor 1A (AVPR1A) in social behavior with the study of autism genetics. AVPR1A mRNA was also recently identified as one of the targets of the Fragile $\mathrm{X}$ mental retardation protein (FMRP). In Kim et al's paper, weak evidence of association was found and several new SNPs were identified in AVPR1A. Given the interaction of the mRNA with FMRP, study of the potential function of the SNPs may require consideration of possible effects on FMRP/AVPR1A mRNA interaction. These findings reveal a new target for future research.

We are delighted that Molecular Psychiatry has taken the lead in publishing some of the most exciting new findings in autism, including both translational research and genetics. Having already established ourselves as a key resource in autism research, we continue to stimulate submissions of new research in this rapidly expanding field.

J Licinio, I Alvarado and M-L Wong UCLA Laboratory of Pharmacogenomics E-mail: licinio@ucla.edu

\section{References}

1 Licinio J, Alvarado I. Mol Psychiatry 2002; 7: 229.

2 Licinio J, Wong M-L. Mol Psychiatry 1999; 4: 317-327. 